\title{
A glass full of optimism: Enrichment effects on cognitive bias in a rat model of depression
}

\author{
Sophie Helene Richter • Anita Schick • Carolin Hoyer • \\ Katja Lankisch • Peter Gass • Barbara Vollmayr
}

Published online: 29 May 2012

(C) Psychonomic Society, Inc. 2012

\begin{abstract}
Investigations of cognitive biases in animals are conceptually and translationally valuable because they contribute to animal welfare research and help to extend and refine our understanding of human emotional disorders, where biased information processing is a critical causal and maintenance factor. We employed the "learned helplessness" genetic rat model of depression in studying cognitive bias and its modification by environmental manipulations. Using a spatial judgment task, responses to ambiguous spatial cues were assessed before and after environmental enrichment to test whether this manipulation would cause an optimistic shift in emotional state. Twenty-four congenitally helpless and nonhelpless male rats were trained to discriminate two different locations, "rewarded" versus "aversive." After successful acquisition of this spatial discrimination, cognitive bias was probed by measuring responses to three ambiguous locations. Latencies to "reach" and to actively "choose" a goal pot were recorded alongside exploratory behaviors. An overall strain difference was observed, with helpless rats displaying longer "reach" latencies than nonhelpless rats. This implies a "pessimistic" response bias in
\end{abstract}

S. H. Richter $(\bowtie) \cdot$ C. Hoyer $\cdot$ K. Lankisch $\cdot$ P. Gass $\cdot$ B. Vollmayr RG Animal Models in Psychiatry,

Department of Psychiatry and Psychotherapy,

Central Institute of Mental Health,

Medical Faculty Mannheim/Heidelberg University,

J5, 68159 Mannheim, Germany

e-mail: helene.richter@zi-mannheim.de

A. Schick

Section for Experimental Psychopathology and Neuroimaging,

Department of General Psychiatry, Center for Psychosocial

Medicine, Heidelberg University,

Heidelberg, Germany helpless rats, underscoring their depressive-like phenotype. No strain differences were observed regarding other behavioral measures. Half of the animals were then transferred to enriched cages and retested. Environmental enrichment resulted in reduced "choose" latencies in both rat strains, associating enrichment with more optimistic interpretations of ambiguous cues. Our results emphasize the suitability of cognitive bias measurement for animal emotion assessment. They extend the methodological repertoire for characterizing complex phenotypes and bear implications for animal welfare research and for the use of animal models in preclinical research.

Keywords Affective state - Cognitive bias - cLH rats . Depression · Environmental enrichment

\section{Introduction}

In humans, it is well established that affective state can influence cognitive processes, a phenomenon referred to as "cognitive bias" (Gotlib \& Joormann, 2010; Mendl, Burman, Parker, \& Paul, 2009). Information can thus be interpreted, attended to, or remembered in such a way that certain types of emotional valence or meaning are favored during processing, making cognitive biases important codeterminants of behavior. Biased information processing and output have been shown to be particularly relevant for depression and anxiety disorder (Mathews \& MacLeod, 2005), where they are taken to be critically contributing causal and maintenance factors (Beck, 1976; Mathews \& Mackintosh, 1998). Regarding the question of whether and how cognitive biases might be therapeutically targeted, 
pharmacological as well as psychological methods have been investigated, and their abilities to modify cognitive biases have indeed been demonstrated (Browning, Holmes, \& Harmer, 2010).

The question of whether nonhuman animals also experience emotions is currently the subject of intense debate. Since concerns for animal welfare are generally based on the assumption that animals can subjectively experience pain or suffering, research focuses on the development of objective methods for the assessment of affective states in animals. Typically, emotional state has been inferred from a variety of different physiological and/or behavioral measures (e.g., Abou-Ismail, Burman, Nicol, \& Mendl, 2007; Burman, Ilyat, Jones, \& Mendl, 2007; Hurst, Barnard, Tolladay, Nevision, \& West, 1999; Mason, Cooper, \& Clarebrough, 2001). Because the relation between affective states and physiological or behavioral measures is nontransitive, the interpretation of such parameters is not always straightforward, and their use as indices of emotional state is limited (Paul, Harding, \& Mendl, 2005). Cognitive components of emotional processing may therefore be a powerful tool to identify unequivocal measures of emotion in animals and, apart from their relevance for animal welfare, could also substantially enhance the use and value of animal models as translational tools in the study of affective dysfunction.

Beginning with the seminal study by Harding, Paul, and Mendl (2004), who introduced a novel approach to determine the emotional states of rats by measuring changes in judgment bias, cognitive bias investigations have been performed in a range of animal species, including rats (Burman, Parker, Paul, \& Mendl, 2008, 2009; Enkel et al., 2010a; Harding et al., 2004), starlings (Brilot, Asher, \& Bateson, 2010; Matheson, Asher, \& Bateson, 2008), dogs (Burman et al., 2011; Mendl et al., 2010), sheep (Doyle, Fisher, Hinch, Boissy, \& Lee, 2010), chicks (Salmeto et al., 2011), rhesus macaques (Bethell, Semple, Holmes, \& MacLarnon, 2007), and even honeybees (Bateson, Desire, Gartside, \& Wright, 2011; Mendl, Paul, \& Chittka, 2011). While the first attempts to develop appropriate tasks for assessing cognitive bias were based on go/no-go decisions (e.g., Bateson \& Matheson, 2007; Harding et al., 2004), the problem of not being able to distinguish between no-go as a response indicator and as a response omission has been overcome by developing go/go tasks, requiring an active response of subjects to both the positive and the negative stimuli (e.g., Brydges, Leach, Nicol, Wright, \& Bateson, 2010; Enkel et al., 2010a; Matheson et al., 2008). Furthermore, a variety of different approaches have been used to manipulate affective state, ranging from unpredictable housing events (Harding et al., 2004) and the provision/removal of environmental enrichment (Brilot et al., 2010; Burman et al., 2008) to the variation of lighting conditions (Burman et al., 2009), and including the induction of both negative (Harding et al., 2004; Salmeto et al., 2011) and positive (Brydges et al., 2010; Burman et al., 2011) emotional states. Notably, there has been a strong emphasis on the study of negative, rather than positive, affective states. This can partly be explained by the current understanding of animal welfare aiming to reduce negative affective states. Moreover, the manifestation of negative experiences may be far more intense, and therefore easier to study, than manifestations of positive states. However, it is now widely accepted that good welfare is both the absence of negative states and the presence of positive experiences (Boissy et al., 2007), making it indispensable to analogously explore positive emotions.

Taken together, investigations of cognitive bias in animals are conceptually and translationally beneficial: First, they contribute to answering pivotal questions relating to welfare issues by providing an unambiguous method of emotion assessment in animals. Second, the modification of cognitive bias in the wake of specific treatments or interventions can furthermore help to extend and refine our understanding of human emotional disorders. This not only concerns pathophysiology and etiology, but may also have implications pertaining to therapy.

On these grounds, we employ a genetic animal model of depression, the "learned helplessness" model (Vollmayr \& Henn, 2001), in the study of cognitive bias and its modification in the rat. Derived from a cognitive theory of human depression (Overmier \& Seligman, 1967) and based on the premise that the experience of uncontrollable stress causes a helpless state with depression-like symptoms, this model possesses a high translational potential for the investigation of a cognitive outcome. By selective breeding of helpless and nonhelpless rats, two strains could be established, congenitally helpless rats (cLH) and rats resistant to helplessness (cNLH; Henn \& Vollmayr, 2005), exhibiting differences in depression-related pathophysiological, neurochemical, and behavioral parameters (Enkel, Spanagel, Vollmayr, \& Schneider, 2010b; Sanchis-Segura, Spanagel, Henn, \& Vollmayr, 2005; Sartorius, Mahlstedt, Vollmayr, Henn, \& Ende, 2007; Vollmayr et al., 2004; Winter, Vollmayr, Djodari-Irani, Klein, \& Sartorius, 2011). Moreover, in a recent study of ambiguous tonal cue interpretation by Enkel, Gholizadeh, et al., 2010a, the depressive-like phenotype of cLH rats was found to manifest in a negative response bias.

In the present study, we aimed to assess whether the "pessimistic" response bias observed in cLH rats also induces a negatively distorted interpretation of ambiguous information in a spatial judgment task (Burman et al., 2009). Using locations instead of auditory tones as the cues, this task taps into rats' natural food-storing and food-retrieval strategies in their burrow-like habitats, and therefore better approximates the animals' ecological situation (Baumans, 2005; Olsson et al., 2003). We further chose to use an 
ecologically relevant mild "punishment"-namely, the "threat" of ingesting aversive food-instead of applying an electric shock as negative reinforcement, since it has previously been argued that the daily experience of punishing events during cognitive bias training may in and of itself affect the state of the animals (Brydges et al., 2010).

We furthermore used environmental enrichment to test whether this form of environmental manipulation leads to an optimistic shift of emotional state, thereby ameliorating depression-like symptoms in our rat model. Environmental enrichment has been shown to modulate stress responses (e.g., Augustsson, Lindberg, Hoglund, \& Dahlborn, 2002; Chourbaji et al., 2008; Olsson \& Dahlborn, 2002; Schrijver, Bahr, Weiss, \& Würbel, 2002), accelerate recovery from a range of clinical conditions (e.g., Buchhold et al., 2007; Gabriel et al., 2010; Pamplona, Pandolfo, Savoldi, Prediger, \& Takahashi, 2009; Percaccio et al., 2005; Vitalo et al., 2012), and induce structural changes in the brain associated with improved cognitive functioning (e.g., Nithianantharajah \& Hannan, 2006; van Praag, Kempermann, \& Gage, 2000). Recently, enrichment has also been used as a tool to induce positive emotional state in tests of cognitive bias in healthy rats (Brydges et al., 2010). By testing background emotional state prior to and after the addition of environmental enrichment to the cages, we sought to test the hypothesis that enrichment would differentially affect judgment biases in helpless and nonhelpless rats.

\section{Animals, materials, and method}

\section{Subjects}

We used 24 males of congenitally helpless (cLH, $n=12$ ) and nonhelpless (cNLH, $n=12$ ) rats from different litters of the 71 st and 72nd generations of the colonies bred at the Central Institute of Mental Health in Mannheim. Originally, both strains had been bred from Sprague-Dawley rats by selecting animals susceptible to helplessness and animals resistant to the effects of uncontrollable stress. All rats had been tested for learned helplessness at the age of 9 weeks. The testing procedures, as well as the origin and selective breeding of these strains, have been described in detail elsewhere (Henn \& Vollmayr, 2005; Vollmayr \& Henn, 2001). Briefly, the test (in boxes from TSE, Bad Soden, Germany) consisted of 15 trials in which an electric foot shock $(0.8 \mathrm{~mA}$, $60 \mathrm{~s}$ ) could be terminated by the animals pressing a bar. Trials not stopped after $20 \mathrm{~s}$ were considered as failures. Animals with nine or more failures were considered to be helpless, while animals with less than five failures were considered to be nonhelpless. The failure patterns of the rats used in the present study ranged from zero to one failures in cNLH rats and from nine to 12 in cLH rats, confirming the nonhelpless versus helpless phenotypes. cNLH rats were exposed to a series of randomized, unpredictable and uncontrollable $0.8 \mathrm{~mA}$ shocks, summing up to a total of $20 \mathrm{~min}, 24 \mathrm{~h}$ prior to the test for learned helplessness.

To test our predictions in a wide range of rats and to avoid unnecessary breeding, we used a heterogeneous group of rats varying in age (22-46 weeks of age at the start of testing), ad libitum feeding body weight (550-700 g), and housing and handling experience prior to testing (handled vs. nonhandled, with different housing densities). To account for this variation in our experimental design, rats were allocated to same-strain cages of two according to these variables. Thus, within a cage, rats were as homogeneous as possible, while rats of different cages varied with respect to these variables. Cage nested within strain was then included in the statistical analysis as a blocking factor, to eliminate variation that would otherwise be assigned to error. By minimizing the error variation, blocking improves the precision of an analysis, thereby avoiding the risk of false positive or negative results. At the same time, using a heterogeneous group of animals increases the external validity of the test outcome and improves reproducibility across experiments (Garner et al., 2011; Richter, Garner, Auer, Kunert, \& Würbel, 2010; Richter, Garner, \& Würbel, 2009; Richter et al., 2011).

The rats were housed in conventional standard macrolon cages (Type IV) with sawdust (Rehofix MK-2000; Rettenmaier \& Söhne, Rosenberg, Germany), additional nesting material in the form of two soft tissue papers, standard rat diet (Ssniff R/M-H; Ssniff Spezialdiäten, Soest, Germany), and tap water ad libitum (= standard housing, $\mathrm{SH}$ ). According to the experimental design, half of the rats were moved to enriched cages 1 week before the second run of cognitive bias testing. The enriched cages (enriched housing $=\mathrm{EH}$ ) additionally contained a gray plastic tunnel $(11 \mathrm{~cm}$ in diameter, $20 \mathrm{~cm}$ long with a bend), two small wooden sticks ("HaselnussKnabberhölzer"; JR Farm, Pessenburgheim, Germany), $200 \mathrm{~g}$ of duff-like bedding material ("Überstreu Waldboden"; Vitakraft, Bremen, Germany), half of a cardboard box $(12 \times$ $12 \times 5 \mathrm{~cm}$ ), and additional shredded tissue papers (Tork Premium, Wash Cloth Soft, Göteborg, Sweden). Moreover, new soft tissue papers were provided daily on the cage lid to keep the rats busy with building a nest. Once per week, the cages were cleaned, water bottles replaced, and black tail markings renewed. The colony room was maintained at a temperature of $22^{\circ} \pm 1{ }^{\circ} \mathrm{C}$, a relative humidity of $50 \% \pm$ $5 \%$, and a 12-h light-dark cycle with the lights off at 19:00.

Depending on the position in the rack, the cages might differ in local environmental conditions (e.g., temperature, lighting, and disturbance) due to variations in proximity to ventilation, lights, and human traffic. To avoid position bias, we controlled for cage position in the experimental design (Herzberg \& Lagakos, 1991). Thus, the 12 cages were 
stacked in four horizontal lines of three cages in one rack, with cages of $\mathrm{cLH}$ and $\mathrm{cNLH}$ rats balanced for horizontal and vertical positions in the rack.

Prior to testing, ad libitum feeding weights were obtained, and rats were then food restricted to $85 \%$ of these initial weights during training and testing. To maintain the animals in a healthy state and to adjust the daily amount of food individually, the weight and health status of each animal were checked on a daily basis. However, since most of the rats were initially overweight, food restriction did not cause any observable changes in the animals' behavior.

All procedures complied with the regulations covering animal experimentation within the EU (European Communities Council Directive 86/609/EEC). They were conducted in accordance with the institutions' animal care and use guidelines and approved by the national and local authorities (Regierungspräsidium Karlsruhe). Moreover, all efforts were made to minimize the number of animals used and the severity of the procedures applied in this study.

\section{Experimental design}

In a repeated parallel design, responses to ambiguous locations and anhedonia-like behavior were assessed in cLH and cNLH rats (Fig. 1). Behavioral testing was done before and after adding enrichment to one half of the cages, separating the experiment in two phases.

Rats were tested in three consecutive replicates $(n=4 /$ strain) separated by a 2 -week interval. Testing was completed for all three replicates before moving to the second phase, leading to a total test time of 12 weeks. One week before the start of the second cognitive bias (CB) run, one cage of two animals per strain and replicate was randomly allocated to either the enriched (EH) or the standard (SH) housing conditions.

\section{Apparatus}

Following the testing protocol introduced by Burman et al. (2009), we used an eight-arm radial maze (arm length $50 \mathrm{~cm}$, arm width $14 \mathrm{~cm}$, hub diameter $40 \mathrm{~cm}$ and height
$40 \mathrm{~cm}$, raised $50 \mathrm{~cm}$ above the floor) made of dark gray Perspex with manually operating guillotine doors separating the central area from each of the eight arms (Fig. 2). The maze was placed in a different testing room close to the colony room, where stable dim light (30 lux) could be provided. The guillotine doors could be opened/closed using a pulley system operated from behind a screen dividing the room in two areas so that the experimenter was not visible to the rats during training and testing.

Only five arms of the maze were used-namely, two reference arms (positive $=$ rewarded, negative $=$ aversive) and three ambiguous locations. The three unused arms were permanently blocked by closed doors (Fig. 2). The two reference arms were positioned $180^{\circ}$ from each other, while the three ambiguous arms were positioned at equidistant angles $\left(45^{\circ}\right)$ between the two reference arms. The ambiguous locations were termed the "near-negative," "near-positive," and "intermediate" arms. As a visual cue within the center of the maze, a piece of colored tape was placed in front of the central unused arm (Fig. 2).

At the beginning of each trial, the appropriate guillotine door was opened to a height of $15 \mathrm{~cm}$, and the rat was allowed to enter the arm. Each arm was partly confined by a tunnel-like plastic cover (cover length $20 \mathrm{~cm}$, cover height $20 \mathrm{~cm}$ ) to prevent the rats from climbing from one open arm to the other instead of entering a new arm out of the center, without reducing the visual perception of the apparatus as a whole. Furthermore, these enclosed parts reduced the aversive character of the arms, facilitating the habituation to the apparatus in the first phase of the experiment. Located $4 \mathrm{~cm}$ from the end of each arm were recessed goal pots (diameter $4 \mathrm{~cm}$, depth $4 \mathrm{~cm}$ ), which could be removed and cleaned between trials.

\section{Cognitive bias}

Procedure We based our protocol on that previously described by Burman et al. (2009), who had not found location-specific treatment effects of variation in lighting conditions on the judgments of ambiguous locations. Assuming that the five locations might have been too easily
Fig. 1 Experimental design. Congenitally helpless (cLH) and nonhelpless (cNLH) rats were tested in a cognitive bias (CB) test followed by measuring the intake of sweetened condensed milk (SCM). Responses to ambiguous locations and SCM intake were assessed before and after adding enrichment (standard housing $=\mathrm{SH}$, enriched housing $=\mathrm{EH}$ ) to half of the cages

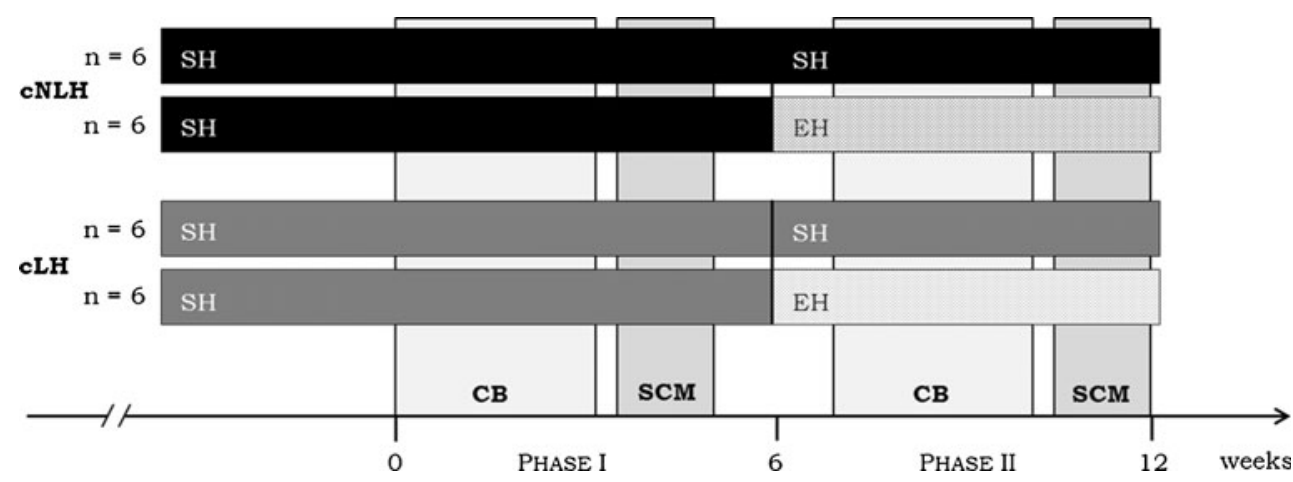




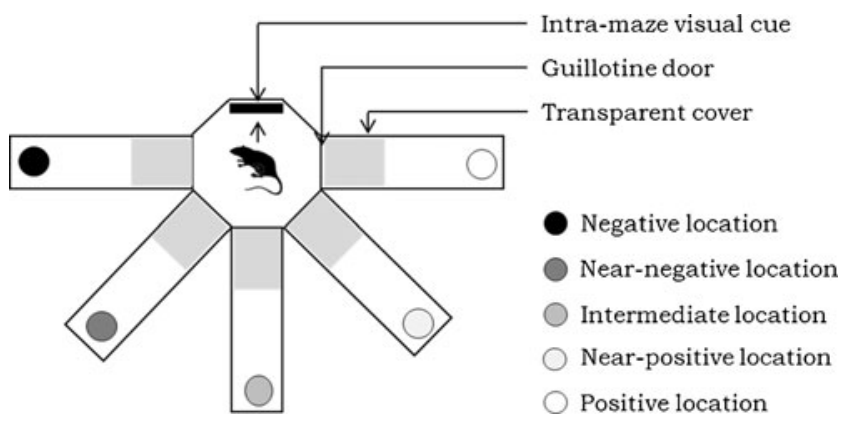

Fig. 2 Experimental apparatus. A schematic diagram of the apparatus shows the two reference locations (negative and positive), the three ambiguous locations, the guillotine doors, the intramaze visual cue, and the transparent covers confining the first $20 \mathrm{~cm}$ of each arm

distinguishable for the rats, we made the following five key modifications:

1. We removed all additional, extramaze cues in the testing room to increase the ambiguous character of the ambiguous locations and make it more difficult for the rats to distinguish between the arms.

2. We trained the rats to reach an individual learning criterion. This enabled us to control for interindividual variation, making sure that individual learning abilities did not interfere with the testing of affective states.

3. We extended the habituation phase to increase the familiarity with the apparatus and reduce neophobiclike behaviors.

4. In addition to the time taken for the nose of the rat to become level with the goal pot, which had previously been used as the key measure for the analysis, we also recorded the latency until the rat put its nose into the goal pot and the numbers of arm entries, head dips, and rearings.

5. Partial reinforcement has previously been shown to slow extinction learning (Bateson \& Matheson, 2007; Brydges et al., 2010; Matheson et al., 2008). We therefore decided not to reward one out of six positive trials carried out each day during discrimination training.

The rats were housed under stable conditions for a minimum of 8 weeks before the onset of the first testing run. The first run consisted of three phases, starting with the habituation to the test apparatus, followed by discrimination training and the final testing phase; for the second run, we skipped the habituation phase, assuming that the apparatus and the procedure were still familiar to the rats. The testing order was randomized on a daily basis, and the rats of one replicate were tested successively. In order to avoid any possible experimenter bias, the whole experiment was run as a blind experiment. At the beginning of each test session, all rats were transported to the test room in their home cages and allowed to acclimatize to the room for $15 \mathrm{~min}$ before testing commenced. The testing was done during the light phase of the cycle.

Habituation Before habituating the rats to the apparatus, we familiarized them with the fruit loops (Fruit Rings, Crownfield, Nordgetreide, Lübeck, Germany) used as rewards by placing six fruit loops in each cage of two rats for three consecutive days. On Day 3 of fruit loop exposure, habituation to the apparatus started. The rats were preexposed to the maze on three consecutive days in order to ensure familiarity with the apparatus, with being enclosed in the center of the maze, and with obtaining fruit loops in the maze. At the beginning, an individual rat was placed in the center of the maze, initially facing the intramaze cue. The rat was then allowed to explore the center for 4 min with all doors closed and 10 half fruit loops scattered on the floor. After $4 \mathrm{~min}$, the five doors were opened simultaneously, and the rats were allowed to explore the whole apparatus for $6 \mathrm{~min}$. We recorded the number of arms visited (defined as a rat placing all four paws in the arm), the number of fecal boli dropped, and the number of fruit loops retrieved.

Training After habituation, the rats were trained to discriminate between the two reference locations. In each training trial, rats had access to either the "positive" (= rewarded) arm, containing four half fruit loops, or to the "negative" (= aversive) arm, containing one quinine-soaked fruit loop. Fruit loops were soaked in quinine by briefly placing them into a $2 \%$ quinine sulphate solution. A training session consisted of 12 trials per day and rat, half positive and half negative. A trial started with opening one door, allowing rats to enter the arm, and ended with the rat actively putting its nose in the goal pot or reaching a cutoff point after $120 \mathrm{~s}$.

The positions of the positive and negative arms were balanced between individuals and strains, with the two rats per cage always being trained to opposite reference arms. The training started with a fixed schedule on Day 1, which was the same for all subjects (sequence: --++--+-+-+- ; see Burman et al., 2009). For the subsequent training days, we used pseudorandomized sequences with no more than two consecutive presentations of the same reference location and equal numbers of both locations in Trials 1-6 and Trials 7 12. Furthermore, Trials 1 and 2 always comprised one rewarded and one aversive trial. To slow extinction learning, one of the three positive trials in Trials 6-12 was not rewarded (Brydges et al., 2010), leading to an overall sequence like $-++--+-+($ not rewarded $)+--+$, with the order of trials being altered daily.

Before each trial, the whole apparatus was cleaned with alcohol (antifect ${ }^{\circledR}$ N liquid; Schülke \& Mayr, Norderstedt, Germany), and the goal pots were placed at the ends of the appropriate arms. The rat was then placed in the center of the maze for a start interval of $1 \mathrm{~min}$ with all arms closed. 
After this minute, the appropriate door was opened, and the time was measured until the rat put its nose in the goal pot at the end of this arm. The rat was then allowed to eat the reward if it chose. Although the positive goal pot contained four half fruit loops, the rat was allowed to retrieve only one before being returned to the center for an intertrial interval (ITI) of $30 \mathrm{~s}$. The number of retrieved rewards was limited to one half fruit loop in order to guarantee that all rats received the same amount of positive experience during training.

The first trial of the first training day was open-ended, while we defined a cutoff point of $120 \mathrm{~s}$ for all of the other trials. If a rat failed to choose the goal pot within this time frame, the rat was put back into the center, and the arena was prepared for the next trial, as usual. Once a rat had completed all 12 training trials, it was put back in the center before we removed it from the maze and prepared the apparatus for the next animal.

To guarantee that the rats were able to reliably discriminate between the two reference locations before being tested, we defined an individual learning criterion. The criterion was reached when a rat showed a significant difference in the latencies to approach the rewarded and aversive goal pots on two consecutive days (Mann-Whitney $U$ Test, onetailed, $p \leq .05$ ). Calculations were based on the latencies recorded from Trial 3 to Trial 12, half of which were rewarded and half not. Each rat was trained for a minimum of 3 days but for no more than 7 days. For the second CB test, discrimination training was limited to a 2-day refresher phase for all rats.

Testing Once a rat had successfully reached the criterion, it was tested on 3 days. A testing session consisted of 13 trials per day per rat. Each ambiguous location was presented once per day, interspersed within a sequence of five rewarded and five aversive trials. The three ambiguous locations were always positioned at Trials 5, 9, and 13, and the order in which the ambiguous locations were presented was counterbalanced over the 3 days. Thus, each ambiguous location was presented three times only, skirting the risk for learning effects associated with repeated presentation of unrewarded ambiguous locations. The overall testing sequence, however, consisted of alternated rewarded and aversive trials, starting either with a positive or a negative trial, so as to correct for the direct effect of a preceding positive or negative experience on the latency to approach an ambiguous location (Burman et al., 2009).

Ambiguous goal pots did not contain any fruit loops during testing. In order to slow association learning between a location and the presence of a reward, the ambiguous arms were presented only once per day. However, since we included partial reinforcement in the training schedule, associations were unlikely to develop over the course of the three testing days.
We recorded two different latency measures per rat and trial: latency to "reach" (the time taken for the nose of the rat to become level with the goal pot; Burman et al., 2009) and latency to "choose" (the time taken for the rat to put its nose into the goal pot). Furthermore, arm entries (the rat placing all four paws on the arm), as well as head dips (the rat dipping its head over the side of the maze while its body remained on the maze) and rearings (the rat standing upright on its hind limbs, with or without touching a wall surface), were counted. A trial ended with the rat "choosing" a goal pot or reaching the cutoff point. "Reach" latencies were thus either lower than or identical to the "choose" latencies.

Sweetened condensed milk intake

To control for differences in reward sensitivity between cLH and cNLH rats and to assess the hedonic value of enrichment, we measured the consummatory response to sweetened condensed milk (SCM), a highly palatable reward for rats. On two consecutive days, cLH and cNLH rats were habituated to single housing in small cages (Type III) that were placed in a separate room. Habituation lasted for $1 \mathrm{~h} /$ day, with the rats remaining completely undisturbed during this time. Overnight, the rats were habituated to sweetened condensed milk (Milchmädchen; Nestlé, Vevey, Switzerland) by replacing one water bottle in the home cage with two bottles, each filled with $40 \mathrm{ml}$ of sweetened condensed milk diluted in water (mixing ratio 1:3). On the test day, rats were placed in the cages to which they had been habituated before and were allowed to acclimatize for $5 \mathrm{~min}$ before being allowed to access the SCM solution freely for $15 \mathrm{~min}$. The amount of liquid consumed by each rat was determined by weighing the bottles and was calculated in relation to the individual body weight.

\section{Data analysis}

All data were analyzed using general linear models (GLMs). To meet the assumptions of parametric analysis, residuals were examined graphically for homoscedasticity and outliers, and using the Kolmogorov-Smirnov and ShapiroWilk tests for normal distribution. When necessary, the raw data were transformed using logarithmic transformations, as indicated in the text below. The specific statistical models used are described in the Results section. Whenever possible, the analyses were blocked by cage nested within strain in order to control for cage effects. Type III sums of squares are used throughout to ensure that all of the results would generalize across the other factors in the model (Garner et al., 2011). All statistical tests were conducted using the software package SPSS (version 17.0 for Windows), and differences were considered to be significant at $p \leq .05$. 


\section{Results}

\section{cLH versus cNLH}

\section{Cognitive bias test}

Habituation For the analysis, we averaged data over the 3 days of habituation for each rat and assessed the effects of strain on the outcome measures by using a GLM with "strain" as a fixed and "cage nested within strain" as a blocking factor. The cLH and cNLH rats did not differ with respect to the numbers of fruit loops retrieved $[F(1,12)=$ $2.812, p>.1]$ and the numbers of arms visited $[F(1,12)=$ $0.004, p>.1]$. However, cNLH rats were found to produce more fecal boli than did cLH rats $[F(1,12)=12.248, p=$ $.004]$, although overall defecation levels were rather low in both strains (Table 1).

Training For the training analysis, the latencies to "choose" the goal pot were averaged for the six rewarded and the six aversive trials for each rat and day. One cNLH rat was excluded from the complete analysis because it failed to approach the goal pots, instead staying in the center of the maze during all training trials. A total of 15 out of the 23 rats learned to discriminate between the locations within 3 days; the remaining animals were trained for $4-5$ days, with one exceptional case being trained for 7 days before reaching criterion. Regarding strain differences, cLH rats tended to learn the criterion faster than did cNLH rats [GLM: $F(1,11)=4.513, p=.057$ ], a difference mainly due to the one cNLH rat that was trained for 7 days.

We also used a repeated measures ANOVA with "strain" as a between-subjects factor, "cage nested within strain" as a blocking factor, and "location" (positive vs. negative) as a within-subjects factor to assess group average performance on each training day. A significant "location" effect was already observed on Day 1 of training $[F(1,11)=$ 53.508, $p<.001$; Fig. 3], showing that rats were able to discriminate between the positive and negative arms from the first day. However, no strain or strain-by-location interaction effects were found during training $(p>.1)$.

Testing Testing was carried out over 3 days, leading to a total of 15 positive, 15 negative, three near-positive, three

Table 1 The average numbers of fruit loops retrieved, arms visited, and fecal boli dropped of cNLH and cLH rats during the habituation phase of the cognitive bias testing

Fruit Loops Retrieved (\#) Arms Visited (\#) Boli Dropped (\#)

\begin{tabular}{llll}
\hline cNLH & $6.46 \pm 0.48$ & $9.22 \pm 0.62$ & $1.89 \pm 0.42$ \\
cLH & $7.78 \pm 0.77$ & $9.28 \pm 0.69$ & $0.69 \pm 0.24$ \\
\hline
\end{tabular}

The data are presented as means \pm standard errors of the means. intermediate, and three near-negative trials per subject. The data per animal and location were averaged and analyzed separately for the ambiguous and reference locations because of the difference between them in the numbers of trials used to calculate the means.

Two different latencies were used to analyze the rats' approach behavior toward the goal pots. In both measures, the average latencies to approach the ambiguous locations were intermediate to those for the reference locations. However, while the latencies to "reach" the goal pot on the ambiguous locations were similar to those of the positive location, the latencies to "choose" the goal pot were nearly as high as those for the negative arm (Fig. 4), indicating that these measures may reflect different behavioral strategies.

To determine whether the rats still accurately discriminated between the reference locations during testing, we used a repeated measures ANOVA with "strain" as a between-subjects factor, "cage nested within strain" as a blocking factor, and "location" (positive vs. negative) as a within-subjects factor. Indeed, we found a highly significant effect of location on the latencies to both "reach" $[F(1,11)=$ 206.426, $p<.001$, Fig. 4a $]$ and "choose" $[F(1,11)=$ $351.005, p<.001$, Fig. 4b] the goal pots, with subjects taking longer on the negative than on the positive reference arm. Moreover, a significant strain effect was observed for both latencies [latency to "reach," $F(1,11)=11.636, p=$ .006 ; latency to "choose," $F(1,11)=6.917, p=.023$ ], showing that cLH rats approached the goal pots faster than did the cNLH rats. This was mainly due to differences in approach latencies on the negative arm, which was statistically confirmed by a significant strain-by-location interaction [latency to "reach," $F(1,11)=18.561, p=.001$; latency to "choose," $F(1,11)=7.536, p=.019]$.

Next, we determined whether or not the rats responded differently to the ambiguous locations and whether this response differed between the strains. Therefore, we used a repeated measures ANOVA with "strain" as a betweensubjects factor, "cage nested within strain" as a blocking factor, and "location" (near-positive, intermediate, or nearnegative) as a within-subjects factor, and included running speed as a covariate in the analysis to account for individual differences in performance. According to Burman and colleagues' (Burman et al., 2008; Burman et al., 2009) method, calculation of the running speed was done for each individual rat by averaging the times taken to "reach" or to "choose" the positive and negative locations. Concerning the latency to "reach" the goal pot, no effect of "location" or "location $\times$ strain" interaction was found $(p>.1)$, but there was a significant main effect of "strain" $[F(1,10)=5.699$, $p=.038$; Fig. 5], with cNLH rats approaching the goal pots faster than cLH rats.

Correcting for running speed in the analysis thus appeared to alter the direction of the strain difference: While 
Fig. 3 Latencies to "choose" the goal pot over the first 3 days of training. Subjects (cNLH, $n=11$; cLH, $n=12$ ) were able to discriminate between the positive and negative locations from the first training day. The data are averaged per strain, location, and day and presented as means \pm standard errors of the means

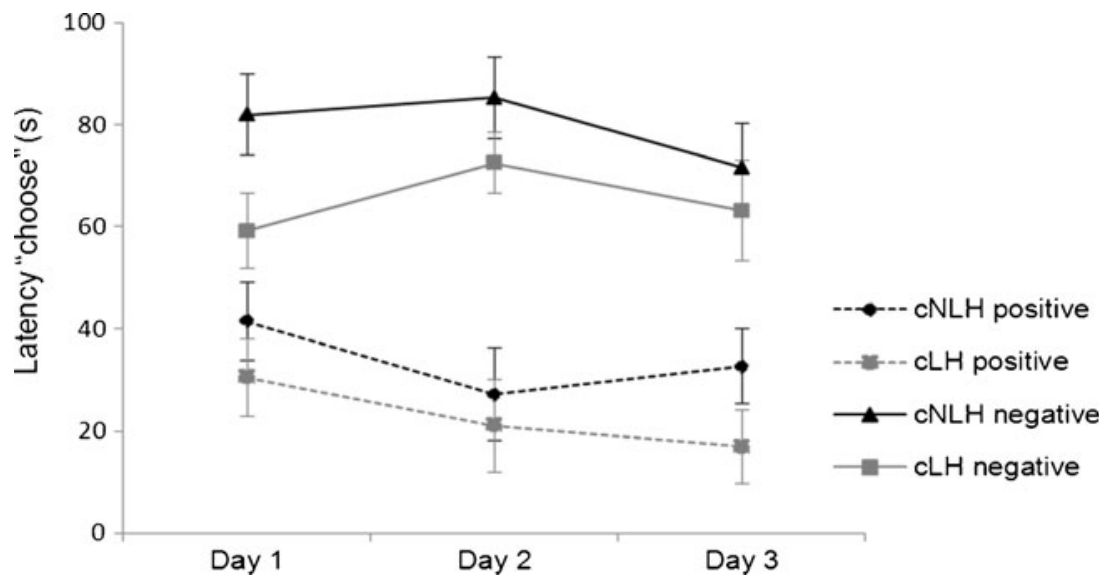

the graphical depiction of the uncorrected latency values (Fig. 4a) suggests a more pessimistic interpretation of ambiguous locations in cNLH rats, evident in longer latencies, the corrected analysis conveys the opposite. To further elaborate on these seemingly conflicting findings, we conducted an additional analysis including the negative location as an intrastrain reference for the interpretation of the ambiguous spatial cues. The negative location was chosen as the intrastrain reference because the latency difference between the strains was more pronounced for this location than for the positive reference arm. We averaged the data across the
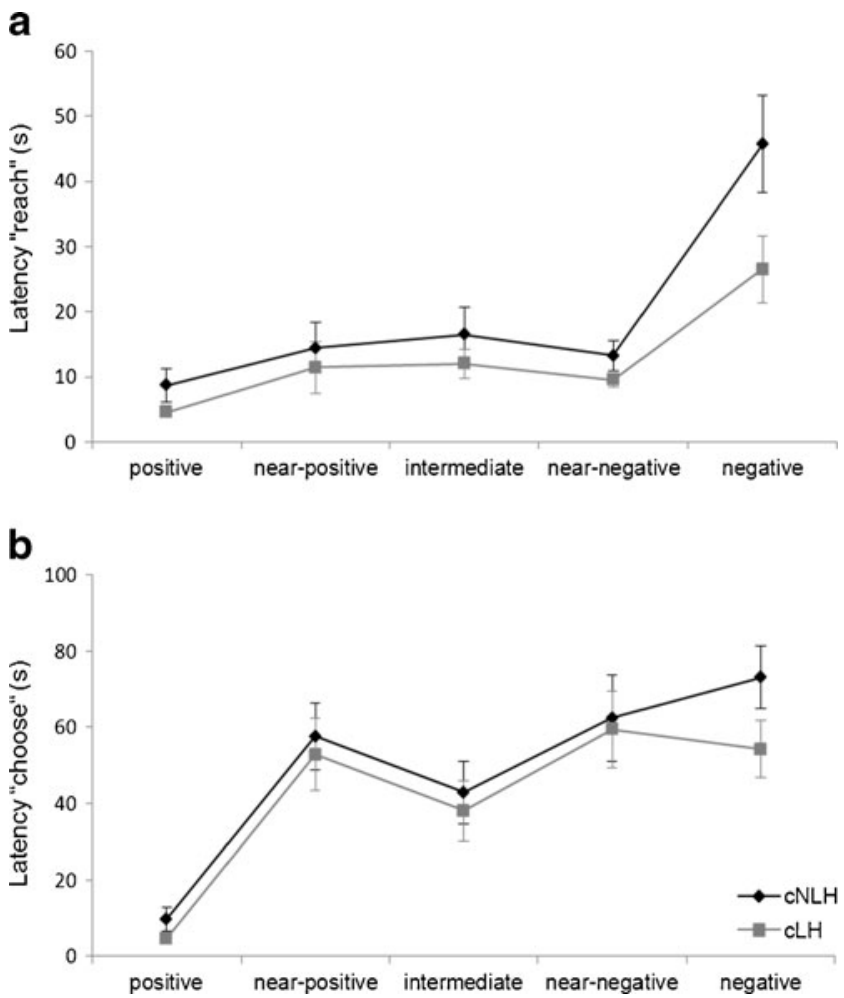

Fig. 4 Latencies to "reach" (a) and "choose" (b) the goal pots on the five arms during testing. The data are averaged per strain and location (cNLH, $n=11 ; \mathrm{cLH}, n=12$ ) and presented as means \pm standard errors of the means three ambiguous arms and performed a repeated measures ANOVA with "strain" as a between-subjects factor, "cage nested within strain" as a blocking factor, and "location" (average ambiguous vs. negative) as a within-subjects factor. Conducting this analysis, significant main effects of "location" $[F(1,11)=161.627, p<.001 ;$ Fig. 6] and "strain" $[F(1,11)=7.937, p=.017$; Fig. 6] were found, showing that the ambiguous arms were approached faster than the reference location and that $\mathrm{cLH}$ rats displayed shorter latencies on both locations. However, the differences in approach latencies between reference and ambiguous arms were far greater in cNLH than in cLH rats, indicating that the relative interpretation of ambiguous cues was more negative in cLH than in cNLH rats. This was statistically confirmed by a significant interaction between "strain" and "location" $[F(1,11)=23.473, p=.001$; Fig. 6].

Concerning the latency to "choose," a significant main effect of "location" was found $[F(2,20)=3.484, p=.05]$, but there was no effect of "strain" and no interaction between "strain" and "location" $(p>.1)$. Thus, the latency to "choose" the goal pot was lowest on the intermediate arm (estimated marginal mean: 38.503 ), followed by the near-

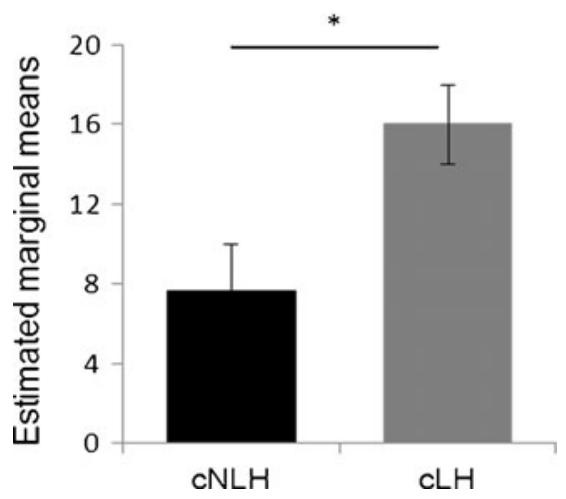

Fig. 5 Differences between cNLH $(n=11)$ and cLH $(n=12)$ rats in latencies to "reach" the goal pots in the three ambiguous arms. The data are presented as estimated marginal means \pm standard errors of the means from the GLM output, in order to account for the use of the covariate "running speed" in the model. ${ }^{*} p<.05$ 


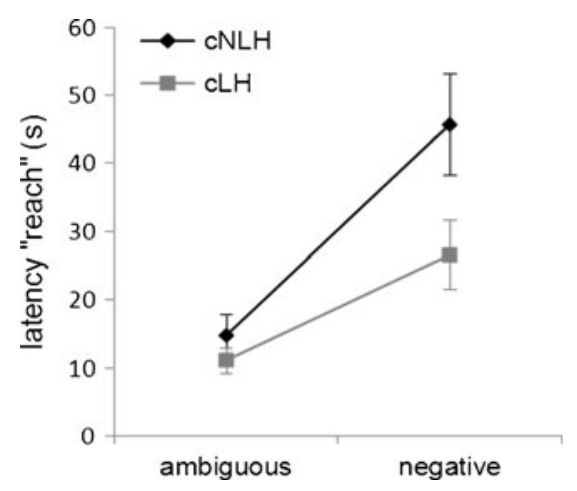

Fig. 6 Differences between cNLH $(n=11)$ and cLH $(n=12)$ rats in latencies to "reach" the goal pots on the ambiguous arms and in the negative reference location. The data of the ambiguous arms are averaged over the three locations and presented as means \pm standard errors of the means

positive (estimated marginal mean: 52.920) and the nearnegative (estimated marginal mean: 63.997) arms.

Next, we analyzed the behavioral measures "rearing," "head dips," and "arm entries" (Table 2). To account for differences in the time needed to complete a trial, the frequencies of rearing and head dipping were divided by the latencies to "choose" a goal pot and then analyzed using a repeated measures ANOVA with "strain" as a betweensubjects factor, "cage nested within strain" as a blocking factor, and "location" (positive, negative, near-positive,

Table 2 Numbers of head dips, rearings, and arm entries of cNLH $(n=11)$ and $\mathrm{cLH}(n=12)$ rats displayed on the two reference (positive and negative) and the three probe (near-positive, intermediate, and near-negative) locations

\begin{tabular}{llll}
\hline & & cNLH & cLH \\
\hline Head dips & Positive & $0.020 \pm 0.005$ & $0.019 \pm 0.006$ \\
& Near-positive & $0.056 \pm 0.008$ & $0.056 \pm 0.010$ \\
& Intermediate & $0.050 \pm 0.009$ & $0.057 \pm 0.013$ \\
& Near-negative & $0.061 \pm 0.011$ & $0.051 \pm 0.006$ \\
Rearings & Negative & $0.031 \pm 0.004$ & $0.043 \pm 0.006$ \\
& Positive & $0.054 \pm 0.025$ & $0.032 \pm 0.020$ \\
& Near-positive & $0.047 \pm 0.014$ & $0.072 \pm 0.025$ \\
& Intermediate & $0.058 \pm 0.007$ & $0.050 \pm 0.014$ \\
& Near-negative & $0.065 \pm 0.006$ & $0.039 \pm 0.008$ \\
& Negative & $0.041 \pm 0.003$ & $0.033 \pm 0.006$ \\
& Positive & $1.045 \pm 0.026$ & $1.012 \pm 0.008$ \\
& Near-positive & $2.030 \pm 0.177$ & $1.999 \pm 0.183$ \\
& Intermediate & $1.878 \pm 0.278$ & $1.666 \pm 0.188$ \\
& Near-negative & $1.908 \pm 0.175$ & $1.875 \pm 0.309$ \\
& Negative & $1.605 \pm 0.122$ & $1.617 \pm 0.117$ \\
& &
\end{tabular}

The absolute values of head dips and rearings are divided by the latencies to "choose" a goal pot, in order to correct for the time needed to complete a trial. The data are presented as means \pm standard errors of the means intermediate, or near-negative) as a within-subjects factor. Analyses were again performed separately for the reference and probe locations. Subjects were found to visit the negative arm more often than the positive one $[F(1,11)=86.312$, $p<.001]$. Furthermore, despite the correction for time spent on an arm, rats were found to perform more head dips on the negative than on the positive $\operatorname{arm}[F(1,11)=7.717$, $p=.018]$, while there was no difference in rearing behavior $(p>.1)$. Concerning the behavior displayed on the ambiguous arms, neither overall effects of "strain" and "location" nor any interaction was observed $(p>.1)$.

\section{Sweetened condensed milk intake}

The amount of liquid consumed was recorded for each rat and calculated in relation to its individual body weight. Using a univariate GLM with "strain" as a fixed factor and "cage nested within strain" as a blocking factor, no difference in milk intakes between cNLH $(23.713 \pm 1.484 \mathrm{~g} / \mathrm{kg})$ and $\operatorname{cLH}(21.585 \pm 1.591 \mathrm{~g} / \mathrm{kg})$ rats was observed $[F(1,12=$ $1.481, p=.247]$.

\section{Enrichment effects}

\section{Cognitive bias test}

Training Prior to the second CB testing, 2 days of refresher training were carried out to ensure that all rats were able to discriminate between the reference locations after a 4-week interval. One more $\mathrm{cNLH}$ rat was excluded from this analysis because it failed to approach the goal pots from the second day of training on, leading to the following sample sizes: $\mathrm{cNLH}$ $\mathrm{SH}=6, \mathrm{cLH} \mathrm{SH}=6, \mathrm{cNLH} \mathrm{EH}=4, \mathrm{cLH} \mathrm{EH}=6$.

A repeated measures ANOVA with "strain" and "housing condition" as between-subjects factors and "location" (positive vs. negative) as a within-subjects factor revealed that rats successfully discriminated between the locations, with significantly longer latencies shown for the negative than for the positive location on both days [Day $1, F(1,18)=$ 251.644, $p<.001$; Day 2, $F(1,18)=395.916, p<.001]$. Furthermore, there was a significant interaction between "strain" and "location" $[F(1,18)=4.834, p=.041]$ and a tendency for standard-housed rats to display higher latencies, as compared to the enriched-housed rats, on Day 1 of refresher training $[F(1,18)=4.250, p=.054]$. This effect was mainly due to a difference between the standard- and enriched-housed cNLH rats, as confirmed by a tendency in the strain-by-housing interaction effect $[F(1,18)=4.150, p=$ .057]. These effects, however, were not observed on Day 2 of refresher training $(p>.1)$.

Testing Using each animal as its own control, we compared behavioral performance between the first and second $\mathrm{CB}$ 
tests. Enrichment-dependent responses to the ambiguous locations were investigated using a repeated measures ANOVA with "strain" and "housing condition" as between-subjects factors and "location" (near-positive, intermediate, or near-negative) and "CB test" (first or second run) as within-subjects factors. To control for the possibility that differences in latencies to probe locations could be due to individual differences between the rats in their running speeds, we adjusted the data using the following equation that had previously been suggested by Burman et al. (2011):

Adjusted score $=($ Mean latency to probe - Mean latency to positive arm $) /($ Mean latency to negative arm - Mean latency to positive arm $)$

This score has been proposed to express all probe test latencies as a proportion of the difference between each rat's baseline mean latencies to the positive and negative reference arms (Burman et al., 2011; Mendl et al., 2010). Moreover, the latency data were log-transformed to meet the assumptions of parametric analysis.

No effects were found on the adjusted scores for the latencies to "reach" the goal pot $(p>.1)$. However, with respect to the adjusted scores for the latencies to "choose" the goal pot, we observed a significant interaction between "housing condition" and "CB test" $[F(1,18)=8.494, p=$ .009] as well as an effect of "location" $[F(2,36)=3.250, p=$ $.05]$, but no overall effects of "strain", "CB test", and "housing condition" ( $p>.1)$. Further investigation of the interaction effect was carried out by analyzing the housing conditions separately. This analysis revealed a significant reduction of "choose" latencies in animals that had been moved to enriched cages $[F(1,4)=8.881, p=.041]$, while there was no difference in latencies between the first and second $\mathrm{CB}$ runs in animals that had remained in standard cages $[F(1,4)=1.657, p=.245$; Fig. 7]. Furthermore, a tendency for a "location" effect was found in $\mathrm{SH}$ rats $[F(2$, $12)=3.411, p=.067]$, while no effects of "strain" and "location" were found in the EH animals $(p>.1)$, indicating that the enrichment effects occurred independently of strain and location.

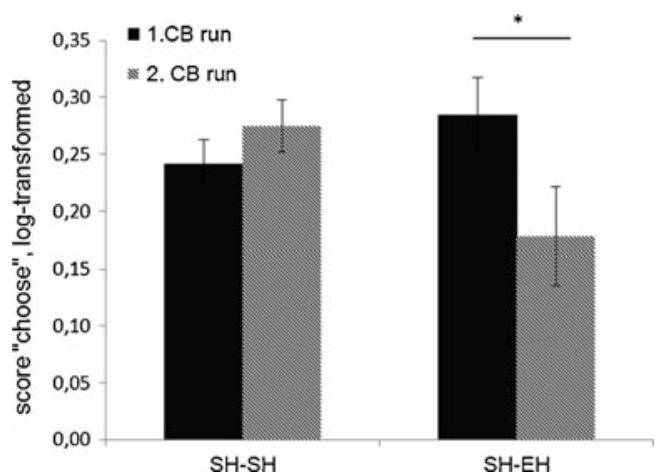

Fig. 7 Scores based on latencies to "choose" the goal pot. The data are averaged across strains and locations and presented as means \pm standard errors of the means from the GLM output. While scores do not differ between the first and second $\mathrm{CB}$ runs for rats housed in standard conditions throughout the experiment (SH-SH), the housing change to enriched conditions (SH-EH) leads to a reduction of score values. ${ }^{*} p<.05$
Concerning the exploratory behaviors shown on the ambiguous arms, no overall housing effects were found on head dips, rearing, and the numbers of arm entries $(p>.1)$. However, we did observe an interaction tendency between "housing condition" and CB run for head dips $[F(1,18=$ $3.508, p=.077]$, in that rats transferred to enriched housing conditions performed relatively more head dips in the second CB run (CB Run 1, $0.055 \pm 0.008$; CB Run 2, $0.066 \pm$ 0.009 ), while animals remaining in standard housing conditions displayed no such changes (CB Run 1, $0.056 \pm$ 0.007; CB Run 2, $0.050 \pm 0.008$ ). In addition, we found effects of $\mathrm{CB}$ run on rearing $[F(1,18)=4.980, p=.039]$ and number of arm entries $[F(1,18)=4.141, p=.057]$, with values in the second $\mathrm{CB}$ run being lower for both parameters. Finally, no effects of "strain" and "location" were found on the exploratory behavioral measures $(p>.1)$.

\section{Sweetened condensed milk intake}

Since the overall intake rate of SCM increased from the first $(22.649 \pm 1.095 \mathrm{~g} / \mathrm{kg})$ to the second $(33.509 \pm 1.992 \mathrm{~g} / \mathrm{kg})$ test in all rats, we analyzed the SCM tests separately using a univariate GLM with the fixed factors "strain" and "housing condition". While there were no effects of "strain" and "housing condition" on milk intake in the first SCM test $(p>.1)$, enriched-housed rats tended to consume more milk than did standard-housed rats in the second $\operatorname{SCM}$ test $[F(1,20)=$ 3.508, $p=.076]$, indicating that enrichment might indeed affect the reward sensitivity. Group comparisons split by strains showed that this effect was present in cNLH $(t$ test for independent data: $t=-2.328, p=.042$ ), but not in cLH $(t=-1.355, p=.205)$, rats in the second SCM test (Fig. 8).

\section{Discussion}

The present study investigated cognitive bias in a spatial judgment task in a genetic rat model of depression, the congenital-learned-helplessness model (Vollmayr \& Henn, 2001), prior to and following environmental enrichment. Our aims were, first, to contribute to the establishment of reliable methods for measuring animals' emotions and, second, to shed light on cognitive aspects of information 


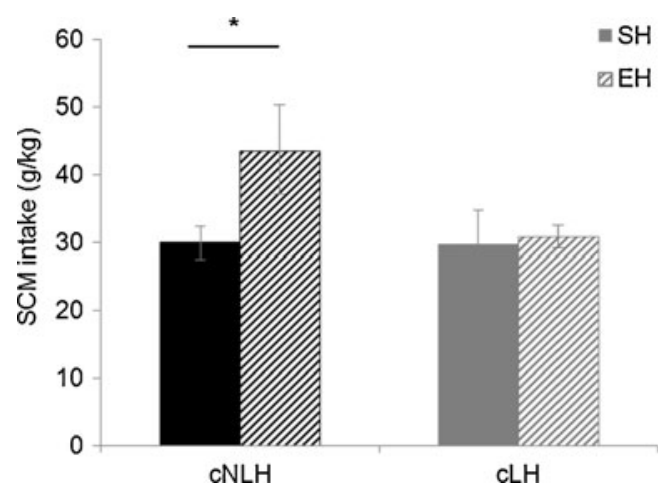

Fig. $8 \mathrm{SCM}$ intake of cNLH and cLH rats housed in standard (cNLH, $n=6$; $\mathrm{cLH}, n=6$ ) or enriched (cNLH, $n=4$; cLH, $n=6$ ) cages. The data are presented as means \pm standard errors of the means for the second SCM test, after transferring half of the animals to enriched cages. ${ }^{*} p<.05$

processing in a state of affective dysfunction. By integrating enrichment into the design, we aimed to further clarify the value of such modifications for animal welfare issues.

Our protocol was based on a judgment bias task developed by Burman et al. $(2008,2009)$. Over the course of the present experiment, rats were housed in 12 stable social groups, half of which were transferred to enriched cages. We chose such a repeated measures design with stable groups to precisely measure individual reactions to enrichment, making the experiment more efficient and allowing for smaller sample sizes (Quinn \& Keough, 2002). Moreover, stable social groups of two animals per cage were used throughout the experiment to avoid disruption due to cagemate changes. Concern has been raised over using the behavior of individuals from one cage as independent measures (Tilly, Dallaire, \& Mason, 2010; Weary \& Fraser, 1998). To account for the fact that the patterns of behavior of individuals from one cage may be more dependent on each other than are those of individuals from different cages, we included cage nested within strain as a factor in the statistical analysis of strain differences (Garner et al., 2011). In comparison to a previous study that analyzed enrichment effects on the basis of just one enriched and one non-enriched cage (Brydges et al., 2010), we based our experiment on three times as many cages. Nonetheless, one may argue that we effectively had a sample size of $n=3$ for testing the effect of enrichment. To counter this interpretation, we follow the argumentation of Brydges et al.: The two housing groups did not differ in any consistent way other than the provision or absence of enrichment. The subjects were tested individually according to a randomized testing order, thereby avoiding confounding effects of social interactions during testing. Furthermore, any modification in social behavior due to the provision of enrichment within a cage can be interpreted as an important part of the enrichment itself.

\section{cNLH versus cLH}

In light of preclinical and clinical evidence for complex interactions between the cognitive and emotional domains (Kalueff \& Murphy, 2007), a methodologically sound approach to cognitive bias assessment requires the exclusion of differences in discrimination acquisition as a confounding factor. By applying an individual learning criterion, the discrimination between the positive and negative reference arms as a key prerequisite for probing ambiguous locations was reliably acquired by both cLH and cNLH rats. Overall, the rats required relatively little time for discrimination acquisition, which for most animals was completed after three days of training. This relatively short training time enhances the procedure's potential for use in behavioral phenotyping and confirms the use of location as an ecologically relevant cue for testing cognitive abilities under laboratory conditions.

A further methodological aspect concerns the interpretation of latencies as exclusively movement-based readouts of our experiment, because those may be confounded by affective state-associated differences in motivation. Alternative approaches to cognitive bias investigation may therefore use non-movement-based readouts - for instance, touchscreen inputs. The use of latencies required the inclusion of the individual running speeds in the analysis. We were surprised, however, to find cNLH rats displaying significantly lower running speeds than cLH rats during the test trials. In a previous study, Vollmayr et al. (2004) noted initial hyperactivity of cLH animals in the open field. Since differences in running speeds between $\mathrm{cLH}$ and $\mathrm{cNLH}$ rats were noticeable from the outset and persisted throughout the testing phase, we are drawn to propose a certain degree of hyperlocomotion as an inherent quality of cLH rats. This contrasts with findings of reduced locomotion both in animal models of depression (Caldecott-Hazard, Mazziotta, \& Phelps, 1988) and clinically (American Psychiatric Association [APA], 2000). Notably, however, agitation is also listed as a diagnostic criterion for depressive disorder (APA, 2000). Moreover, resistance to habituation, increased anxiety (Schulz, Mirrione, \& Henn, 2010), and higher levels of stress or arousal in cLH rats may be invoked as explanations for these observations.

Despite a strain effect regarding running speeds, there were no differences in exploratory behavior between the $\mathrm{cLH}$ and cNLH rats, indicating that our strains were not characterized by general differences in risk assessment and novelty-induced behaviors (File \& Wardill, 1975; Walf \& Frye, 2007). Furthermore, exploratory behavior did not differ between the three ambiguous arms, indicating that these locations were all interpreted in somewhat similar manners. However, regarding the reference arms, rats performed more head dips on the negative arm than on the positive arm, 
although the frequencies were corrected for the time needed to complete a trial. Accordingly, rats also visited the negative arm more often than the positive one. Together, this may argue for more goal-oriented behavior on the positive arm, where rats' behavior was clearly motivated by obtaining a food reward. Its absence on the negative arm, however, allowed rats to follow their natural tendency to explore open spaces. This manifests in higher amounts of arm entries and exploratory behavior, thereby further confirming the animals' ability to clearly discriminate between these two locations.

One major finding of our experiment concerns the interpretation of ambiguous locations by cLH and cNLH rats. In the analysis corrected for running speed, $\mathrm{cNLH}$ rats were found to approach ambiguous goal pots faster than cLH rats did, indicating a more negative interpretation of ambiguous spatial cues by cLH rats. This becomes particularly evident when including the negative arm as an intrastrain reference in the analysis, showing that, in relation to the negative arm, the ambiguous locations were interpreted more negatively in cLH than in cNLH rats. The absence of a strain effect in exploratory behavior supports the view that this latency difference is indeed grounded in a negative interpretation bias rather than in a differential perception of novelty. Accordingly, our results corroborate the findings of Enkel et al. (2010a) and confirm the cLH depressive phenotype.

However, while Enkel et al. (2010a) demonstrated a graded response to ambiguous cues, in that animals further differentiated between near-positive, intermediate, and nearnegative, we observed the set of ambiguous spatial locations to be uniformly evaluated. This could mean that a certain degree of novelty associated with ambiguous locations partly overrides a bias-based attribution of emotional valence. Alternatively, there might be differences in the brain's resolution capacities for auditory/frequency stimuli versus spatial/locational information (Burn, 2008; Talwar \& Gerstein, 1998). Interestingly, the same phenomenon was observed by Burman et al. (2009), who also reported an overall treatment effect inclusive of all three ambiguous locations, without any location-specific differences. Since reference and ambiguous arms were viewed differently, as indicated by differences in latencies and exploratory behaviors, the rats seemed able to clearly distinguish between the five arms. To measure cognitive bias, however, an intuition-guided interpretation of ambiguous locations is desirable, whereby the distinction between the reference and ambiguous locations becomes somewhat blurred. Locations on a radial arm-maze may therefore be too easily distinguishable to the rats, which would argue for a more open test arena, as was previously used (Burman et al., 2008). A modification of the test procedure or apparatus may thus contribute further to improving the sensitivity of the entire test procedure.
Unlike Burman et al. (2009), we extended latency measurement by a second parameter, namely the latency to actively "choose" a goal pot. In this regard, a strain effect indicative of a negative bias toward ambiguous locations in cLH rats was present for the latency to "reach" the goal pot, but not for the latency to "choose." Moreover, for "reach," ambiguous arms were approached with latencies similar to those for the positive arm during testing, while latencies to "choose" were longer and more closely approximated values for the negative arm, which may be indicative of a differential influence of sensory input-for instance, olfactory or visual — on the two latency measures. Furthermore, a location effect was observed on the latency to "choose," with the highest latency values for the near-negative location, supporting the feasibility of the test principle. On these grounds, it is reasonable to assume that these measures reflect two distinct behavioral strategies: We suggest that "reach" represents decision-making with respect to the initiation of behavior, and thus possesses a stronger incentive component than does "choosing," which in turn may more strongly reflect outcome-oriented decision-making.

Postulating the coordinative impacts of an incentivebased behavioral strategy ("reach") alongside a more goaldirected one ("choosing") in shaping the final behavioral outcome raises the critical methodological issue of whether the readout of our paradigm - and eventually that of every reward-based paradigm investigating cognitive bias in models of depression - is truly the effect of a cognitive bias on behavior, or rather reflects differences in motivation for behavior rooted in blunted reward sensitivity. SCM intake is a measure employed to assess reward sensitivity and may thus be helpful in answering this question. We recorded no difference in SCM consumption between cNLH and cLH rats. However, intake of sweet solutions is apparently reduced in cLH rats under conditions of stress (Enkel, Gholizadeh, et al., 2010a; Enkel, Spanagel, et al., 2010b; Sanchis-Segura et al., 2005), accentuating the animals' vulnerability to depression-like behavior in adverse circumstances.

Yet, in light of the anatomical and functional points of convergence for affect, motivation, reward sensitivity and high-level cognition, we would like to briefly put forward some aspects pertinent to bias testing and the animal model under scrutiny: Reduced dopaminergic neurotransmission is considered a key contributing factor for depression pathophysiology and phenomenology (Anisman \& Matheson, 2005; Martin-Soelch, 2009). One may postulate the existence of a predictive process, mediated by various midbrain structures, regarding an expected outcome value on the basis of previous experiences (Ji \& Shepard, 2007). This, in turn, suggests a close interrelation of the motivational and cognitive domains, wherein a negative cognitive bias may well influence reward predictions and reward sensitivity. Thus, the question may not be whether we are measuring the 
output of one domain or another, but rather whether the data may prompt us to inquire into the nature of the relationship between these domains.

\section{Enrichment effects}

Enrichment - the addition of inanimate structures to cages aims at facilitating species-specific behaviors (Baumans, 2005; Olsson et al., 2003). It is well established that the increased sensory and motor stimulation provided by environmental enrichment induces structural changes in the rodent brain that are in turn associated with improved cognitive functioning (reviewed by van Praag et al., 2000). However, the effects of environmental enrichment on affective state have been investigated far less intensely and remain more questionable. While some studies have found no or inconsistent effects on emotional reactivity (e.g., Fernandez-Teruel, Escorihuela, Castellano, Gonzalez, \& Tobena, 1997; Lin, Choi, Liu, Martin, \& During, 2011), others have observed a reduction of emotionality in enriched-housed animals (e.g., Chamove, 1989; Manosevitz \& Joel, 1973).

In our experiment, transferring animals into an enriched environment resulted in reduced latencies to actively "choose" a goal pot. Within the conceptual framework of this experiment, this finding implies a more optimistic interpretation of the ambiguous locations that is brought about by enrichment. The increase in optimism was observed in both cNLH and cLH rats, but only with respect to one latency measure. Environmental enrichment has been demonstrated to exert robust improvements on cognition such as spatial learning and memory (Rampon et al., 2000; van Praag et al., 2000; Wainwright, Lévesque, Krempulec, Bulman-Fleming, \& McCutcheon, 1993; Winocur, 1998). An effect of enrichment on latencies to "choose" but not to "reach" corroborates this evidence further, since "choosing" is a behavioral parameter supposed to possess a stronger cognitive component than the incentive-rooted "reach." We further observed a tendency for increased head dips as a manifestation for exploratory behavior in rats transferred to enriched housing conditions. This finding can be considered to reflect increased exploratory drive, and as such a wider species-specific behavioral repertoire, brought about by enrichment (Baumans, 2005).

We did not find a strain-specific effect of environmental enrichment, which at first sight is a surprising finding, as one may have expected a differential response to enrichment by cNLH and cLH rats, given the resistance of cLH animals to electroconvulsive or antidepressant drug treatment (Sartorius et al., 2007). However, cLH rats do respond to environmental enrichment, and there is further evidence for beneficial effects of enrichment in other antidepressant-resistant animal models (Hendriksen, Prins, Olivier, \& Oosting, 2010). This entails an at least partial divergence of the "antidepressant" modes of action of enrichment and, for example, antidepressant drug treatments in animal models (Bjatmar et al., 2010). On the other hand, the overall tendency for increased milk consumption in enriched-housed rats in the second SCM test was mainly due to cNLH rats consuming more milk, while cLH animals' milk consumption remained unaltered. In sum, while a shift toward a more optimistic interpretation of ambiguous spatial cues was observed in both strains, reward sensitivity was only influenced in cNLH rats. These data suggest differential effects of enrichment (Chapillon, Manneche, Belzung, \& Caston, 1999; Marashi, Barnekow, Ossendorf, \& Sachser, 2003; Van de Weerd, Baumans, Koolhaas, \& Van Zutphen, 1994), which in our scenario apply to cognitive appraisal and reward sensitivity: While enrichment brought about a change in the former in "treatment-resistant" animals, it remained without effect regarding the latter.

From a welfare perspective, the induction of a positive processing bias toward an ecologically relevant cue through enrichment underscores the pivotal importance of speciesappropriate conditions of animal keeping that provide adequate sensory and motor stimulation. Our results add to previous work in laboratory animals, showing that the removal of enrichment typically leads to more pessimistic cognitive biases (Bateson \& Matheson, 2007; Burman et al., 2008), while rats were found to respond more optimistically to an ambiguous stimulus when moved to an enriched environment (Brydges et al., 2010; Burman et al., 2008).

Although they involve methodologically different approaches, our own work and the previous study of Brydges et al. (2010) possess some basic methodological commonalities regarding enrichment. In both studies, rats were housed in a barren environment for several weeks before being transferred to enriched cages. Cognitive bias testing was done one week after the housing change in both experiments, raising the question of whether the observed positive bias was a reflection either of the improved housing conditions per se or of the novelty associated therewith. Housing systems are often enriched through the addition of objects and resources, with the aim of ensuring a state of improved welfare. However, since enrichment generally involves both environmental complexity and novelty, it is currently unclear what kind of enrichment is necessary to maintain animals in a state of good welfare (Gross, Engel, \& Würbel, 2011). Although our findings support the general welfare benefits afforded by the provision of enrichment, we cannot dissociate the effects of environmental complexity and novelty on the basis of this study. By testing cognitive bias after long-term enrichment, future studies should thus evaluate whether rats housed in an enriched environment will remain in a state of positive affect or will become bored again after being housed in an enriched environment for several weeks, thereby shedding light on how enrichment acts on welfare measures. 
Summary and conclusion

With our study of cognitive bias in the rat learned-helplessness model, we have demonstrated the presence of a negative processing bias for ambiguous locations in $\mathrm{CLH}$ rats, thereby confirming the depressive-like phenotype of these animals. Through the addition of a cognitive component to existing findings pertaining to pathophysiological, neurobiochemical, and behavioral alterations in cLH rats, our results thus underscore the translational value of the congenital-learnedhelplessness model as a model for depression. By supplying a method for emotion assessment that is ecologically meaningful, and thus important for animal welfare research, but also translationally valuable, we further aimed to establish and refine methods for the assessment of animals' emotions. Finally, the demonstration of the impact of environmental enrichment emphasizes the relevance of adequate and appropriate conditions for animal handling and keeping.

Author note This work was funded through a grant to P.G. and B.V. from the German Ministry of Education and Research (BMBF, 01GQ1003B). Furthermore, we thank Daniel Durstewitz for fruitful discussions about the statistical analyses, and Helene Schamber and Peter Siegel for excellent technical support and animal care.

\section{References}

Abou-Ismail, U. A., Burman, O., Nicol, C. J., \& Mendl, M. (2007). Can sleep behaviour be used as an indicator of stress in grouphoused rats (Rattus norvegicus)? Animal Welfare, 16, 185-188.

Anisman, H., \& Matheson, K. (2005). Stress, depression, and anhedonia: Caveats concerning animal models. Neuroscience and Biobehavioral Reviews, 29, 525-546.

American Psychiatric Association (2000). Diagnostic and statistical manual of mental disorders (4th ed., Text rev.). Washington, DC: Author.

Augustsson, H., Lindberg, L., Hoglund, A. U., \& Dahlborn, K. (2002). Human-animal interactions and animal welfare in conventionally and pen-housed rats. Laboratory Animals, 36, 271-281.

Bateson, M., Desire, S., Gartside, S. E., \& Wright, G. A. (2011). Agitated honeybees exhibit pessimistic cognitive biases. Current Biology, 21, 1070-1073.

Bateson, M., \& Matheson, S. M. (2007). Performance on a categorisation task suggests that removal of environmental enrichment induces "pessimism" in captive European starlings (Sturnus vulgaris). Animal Welfare, 16, 33-36.

Baumans, V. (2005). Environmental enrichment for laboratory rodents and rabbits: Requirements of rodents, rabbits, and research. ILAR Journal, 46, 162-170.

Beck, A. T. (1976). Cognitive therapy and the emotional disorders. New York: International University Press.

Bethell, E. J., Semple, S., Holmes, A., \& MacLarnon, A. (2007). The effect of emotion state on responses to social stimuli by rhesus macaques. Primate Eye, 92, 5-6.

Bjatmar, L., Alkhori, L., Ruud, J., Mohammed, A. H., Marcusson, J., \& Hallbeck, M. (2010). Long-term treatment with antidepressants, but not environmental stimulation, induces expression of NP2 mRNA in hippocampus and medial habenula. Brain Research, 1328, 25-33.
Boissy, A., Manteuffel, G., Jensen, M. B., Moe, R. O., Spruijt, B., Keeling, L. J., ... Aubert, A. (2007). Assessment of positive emotions in animals to improve their welfare. Physiology and Behavior, 92, 375-397.

Brilot, B. O., Asher, L., \& Bateson, M. (2010). Stereotyping starlings are more "pessimistic". Animal Cognition, 13, 721-731.

Browning, M., Holmes, E. A., \& Harmer, C. J. (2010). The modification of attentional bias to emotional information: A review of the techniques, mechanisms, and relevance to emotional disorders. Cognitive, Affective, \& Behavioral Neuroscience, 10, 8-20. doi:10.3758/CABN.10.1.8

Brydges, N. M., Leach, M., Nicol, K., Wright, R., \& Bateson, M. (2010). Environmental enrichment induces optimistic bias in rats. Animal Behaviour, 81, 169-175.

Buchhold, B., Mogoanta, L., Suofu, Y., Hamm, A., Walker, L., Kessler, C., \& Popa-Wagner, A. (2007). Environmental enrichment improves functional and neuropathological indices following stroke in young and aged rats. Restorative Neurology and Neuroscience, 25, 467-484.

Burman, O., Ilyat, A., Jones, G., \& Mendl, M. (2007). Ultrasonic vocalizations as indicators of welfare for laboratory rats (Rattus norvegicus). Applied Animal Behaviour Science, 104, 116129.

Burman, O., McGowan, R., Mendl, M., Norling, Y., Paul, E., Rehn, T., \& Keeling, L. J. (2011). Using judgement bias to measure positive affective state in dogs. Applied Animal Behaviour Science, 132, $160-168$

Burman, O., Parker, R., Paul, E. S., \& Mendl, M. (2008). A spatial judgement task to determine background emotional state in laboratory rats, Rattus norvegicus. Applied Animal Behaviour Science, 76, 801-809.

Burman, O., Parker, R., Paul, E. S., \& Mendl, M. T. (2009). Anxietyinduced cognitive bias in non-human animals. Physiology and Behavior, 98, 345-350.

Burn, C. O. (2008). What is it like to be a rat? Rat sensory perception and its implications for experimental design and rat welfare. Applied Animal Behaviour Science, 112, 1-32.

Caldecott-Hazard, S., Mazziotta, J., \& Phelps, M. (1988). Cerebral correlates of depressed behavior in rats, visualized using 14C-2deoxyglucose autoradiography. Journal of Neuroscience, 8, 1951-1961.

Chamove, A. S. (1989). Cage design reduces emotionality in mice. Laboratory Animals, 23, 215-219. doi:10.1258/002367789780810608

Chapillon, P., Manneche, C., Belzung, C., \& Caston, J. (1999). Rearing environmental enrichment in two inbred strains of mice: 1. Effects on emotional reactivity. Behavior Genetics, 29, 41-46.

Chourbaji, S., Brandwein, C., Vogt, M. A., Dorman, C., Hellweg, R., \& Gass, P. (2008). Nature vs. nurture: Can enrichment rescue the behavioral phenotype of BDNF heterozygous mice? Behavioural Brain Research, 192, 254-258.

Doyle, R. E., Fisher, A. D., Hinch, G. N., Boissy, A., \& Lee, C. (2010). Release from restraint generates a positive judgment bias in sheep. Applied Animal Behaviour Science, 122, 28-34.

Enkel, T., Gholizadeh, D., von Bohlen und Halbach, O., SanchisSegura, C., Hurlemann, R., Spanagel, R., ... Vollmayr, B. (2010a). Ambiguous-cue interpretation is biased under stressand depression-like states in rats. Neuropsychopharmacology, $35,1008-1015$.

Enkel, T., Spanagel, R., Vollmayr, B., \& Schneider, M. (2010b). Stress triggers anhedonia in rats bred for learned helplessness. Behavioural Brain Research, 209, 183-186.

Fernandez-Teruel, A., Escorihuela, R. M., Castellano, B., Gonzalez, B., \& Tobena, A. (1997). Neonatal handling and environmental enrichment effects on emotionality, novelty/reward seeking, and age-related cognitive and hippocampal impairments: Focus on the Roman rat lines. Behavior Genetics, 27, 513-526. 
File, S. E., \& Wardill, A. G. (1975). Validity of head-dipping as a measure of exploration in a modified hole-board. Psychopharmacologia, 44, 53-59.

Gabriel, A. F., Paoletti, G., Della Seta, D., Panelli, R., Marcus, M. A., Farabollini, F., ... Joosten, E. A. (2010). Enriched environment and the recovery from inflammatory pain: Social versus physical aspects and their interaction. Behavioural Brain Research, 208, 90-95.

Garner, J. P., Thogerson, C. M., Dufour, B. D., Würbel, H., Murray, J. D., \& Mench, J. A. (2011). Reverse-translational biomarker validation of abnormal repetitive behaviors in mice: An illustration of the 4P's modeling approach. Behavioural Brain Research, 219, 189-196.

Gotlib, I. H., \& Joormann, J. (2010). Cognition and depression: Current status and future directions. Annual Review of Clinical Psychology, 6, 285-312.

Gross, A. N., Engel, A. K. J., \& Würbel, H. (2011). Simply a nest? Effects of different enrichments on stereotypic and anxiety-related behaviour in mice. Applied Animal Behaviour Science, 134, 239-245.

Harding, E. J., Paul, E. S., \& Mendl, M. (2004). Animal behaviour: Cognitive bias and affective state. Nature, 427, 312. doi:10.1038/ $427312 \mathrm{a}$

Hendriksen, H., Prins, J., Olivier, B., \& Oosting, R. S. (2010). Environmental enrichment induces behavioral recovery and enhanced hippocampal cell proliferation in an antidepressantresistant animal model for PTSD. PLoS One, 5, e11943.

Henn, F. A., \& Vollmayr, B. (2005). Stress models of depression: Forming genetically vulnerable strains. Neuroscience and Biobehavioral Reviews, 29, 799-804.

Herzberg, A. M., \& Lagakos, S. W. (1991). Cage allocation designs for rodent carcinogenicity experiments. Environmental Health Perspectives, 96, 199-202.

Hurst, J. L., Barnard, C. J., Tolladay, U., Nevision, C. M., \& West, C. D. (1999). Housing and welfare in laboratory rats: Effects of cage stocking density and behavioural predictors of welfare. Animal Behaviour, 58, 563-586.

Ji, H., \& Shepard, P. D. (2007). Lateral habenula stimulation inhibits rat midbrain dopamine neurons through a GABA(A) receptormediated mechanism. Journal of Neuroscience, 27, 6923-6930. doi:10.1523/JNEUROSCI.0958-07.2007

Kalueff, A. V., \& Murphy, D. L. (2007). The importance of cognitive phenotypes in experimental modeling of animal anxiety and depression. Neural Plasticity, 2007(52087), 1-7. doi:10.1155/ 2007/52087

Lin, E. J., Choi, E., Liu, X., Martin, A., \& During, M. J. (2011). Environmental enrichment exerts sex-specific effects on emotionality in C57B1/6J mice. Behavioural Brain Research, 216, 349-357.

Manosevitz, M., \& Joel, U. (1973). Behavioral effects of environmental enrichment in randomly bred mice. Journal of Comparative and Physiological Psychology, 85, 373-382.

Marashi, V., Barnekow, A., Ossendorf, E., \& Sachser, N. (2003). Effects of different forms of environmental enrichment on behavioral, endocrinological, and immunological parameters in male mice. Hormones and Behavior, 43, 281-292.

Martin-Soelch, C. (2009). Is depression associated with dysfunction of the central reward system? Biochemical Society Transactions, 37, 313-317.

Mason, G. J., Cooper, J., \& Clarebrough, C. (2001). Frustrations of fur-farmed mink. Nature, 410, 35-36.

Matheson, S. M., Asher, L., \& Bateson, M. (2008). Larger, enriched cages are associated with "optimistic" response biases in captive European starlings (Sturnus vulgaris). Applied Animal Behaviour Science, 109, 374-383.

Mathews, A., \& Mackintosh, B. (1998). A cognitive model of selective processing in anxiety. Cognitive Therapy and Research, 22, 539560 .
Mathews, A., \& MacLeod, C. (2005). Cognitive vulnerability to emotional disorders. Annual Review of Clinical Psychology, 1, 167-195.

Mendl, M., Brooks, J., Basse, C., Burman, O., Paul, E., Blackwell, E., \& Casey, R. (2010). Dogs showing separation-related behaviour exhibit a "pessimistic" cognitive bias. Current Biology, 20, R839R840. doi:10.1016/j.cub.2010.08.030

Mendl, M., Burman, O., Parker, R., \& Paul, E. (2009). Cognitive bias as an indicator of animal emotion and welfare: Emerging evidence and underlying mechanisms. Applied Animal Behaviour Science, $118,161-181$.

Mendl, M., Paul, E. S., \& Chittka, L. (2011). Animal behaviour: Emotion in invertebrates? Current Biology, 21, R463-R465. doi:10.1016/j.cub.2011.05.028

Nithianantharajah, J., \& Hannan, A. J. (2006). Enriched environments, experience-dependent plasticity and disorders of the nervous system. Nature Reviews Neuroscience, 7, 697-709.

Olsson, I. A., \& Dahlborn, K. (2002). Improving housing conditions for laboratory mice: A review of "environmental enrichment. Laboratory Animals, 36, 243-270.

Olsson, I. A., Nevision, C. M., Patterson-Kane, E. G., Sherwin, C. M., Van de Weerd, H. A., \& Würbel, H. (2003). Understanding behaviour: The relevance of ethological approaches in laboratory animal science. Applied Animal Behaviour Science, 81, 245-264.

Overmier, J. B., \& Seligman, M. E. (1967). Effects of inescapable shock upon subsequent escape and avoidance responding. Journal of Comparative and Physiological Psychology, 63, 28-33.

Pamplona, F. A., Pandolfo, P., Savoldi, R., Prediger, R. D., \& Takahashi, R. N. (2009). Environmental enrichment improves cognitive deficits in spontaneously hypertensive rats (SHR): Relevance for attention deficit/hyperactivity disorder (ADHD). Progress in Neuro-Psychopharmacology \& Biological Psychiatry, 33, 1153-1160.

Paul, E. S., Harding, E. J., \& Mendl, M. (2005). Measuring emotional processes in animals: The utility of a cognitive approach. Neuroscience and Biobehavioral Reviews, 29, 469-491.

Percaccio, C. R., Engineer, N. D., Pruette, A. L., Pandya, P. K., Moucha, R., Rathbun, D. L., \& Kilgard, M. P. (2005). Environmental enrichment increases paired-pulse depression in rat auditory cortex. Journal of Neurophysiology, 94, 3590-3600.

Quinn, J. P., \& Keough, M. J. (2002). Experimental design and data analysis for biologists. Cambridge: Cambridge University Press.

Rampon, C., Tang, Y., Goodhouse, J., Shimizu, E., Kyin, M., \& Tsien, J. Z. (2000). Enrichment induces structural changes and recovery from nonspatial memory deficits in CA1 NMDAR1-knockout mice. Nature Neuroscience, 3, 205-206.

Richter, S. H., Garner, J. P., Auer, C., Kunert, J., \& Würbel, H. (2010). Systematic variation improves reproducibility of animal experiments. Nature Methods, 7, 167-168. doi:10.1038/nmeth0310-167

Richter, S. H., Garner, J. P., \& Würbel, H. (2009). Environmental standardization: Cure or cause of poor reproducibility in animal experiments? Nature Methods, 6, 257-261.

Richter, S. H., Garner, J. P., Zipser, B., Lewejohann, L., Sachser, N., Touma, C., ... Würbel, H. (2011). Effect of population heterogenization on the reproducibility of mouse behavior: A multilaboratory study. PLoS ONE, 6, e16461.

Salmeto, A. L., Hymel, K. A., Carpenter, E. C., Brilot, B. O., Bateson, M., \& Sufka, K. J. (2011). Cognitive bias in the chick anxietydepression model. Brain Research, 1373, 124-130.

Sanchis-Segura, C., Spanagel, R., Henn, F. A., \& Vollmayr, B. (2005). Reduced sensitivity to sucrose in rats bred for helplessness: A study using the matching law. Behavioural Pharmacology, 16, 267-270.

Sartorius, A., Mahlstedt, M. M., Vollmayr, B., Henn, F. A., \& Ende, G. (2007). Elevated spectroscopic glutamate/gamma-amino butyric acid in rats bred for learned helplessness. Neuroreport, 18, 1469-1473. 
Schrijver, N. C., Bahr, N. I., Weiss, I. C., \& Würbel, H. (2002). Dissociable effects of isolation rearing and environmental enrichment on exploration, spatial learning and HPA activity in adult rats. Pharmacology Biochemistry and Behavior, 73, 209-224.

Schulz, D., Mirrione, M. M., \& Henn, F. A. (2010). Cognitive aspects of congenital learned helplessness and its reversal by the monoamine oxidase (MAO)-B inhibitor deprenyl. Neurobiology of Learning and Memory, 93, 291-301.

Talwar, S. K., \& Gerstein, G. L. (1998). Auditory frequency discrimination in the white rat. Hearing Research, 126, 135-150.

Tilly, S. C., Dallaire, J., \& Mason, G. J. (2010). Middle-aged mice with enrichment-resistant stereotypic behaviour show reduced motivation for enrichment. Animal Behaviour, 80, 363-373.

Van de Weerd, H. A., Baumans, V., Koolhaas, J. M., \& Van Zutphen, L. F. M. (1994). Strain-specific behavioural response to envinronmental enrichment in the mouse. Journal of Experimental Animal Science, 36, 117-127.

van Praag, H., Kempermann, G., \& Gage, F. H. (2000). Neural consequences of environmental enrichment. Nature Reviews Neuroscience, 1, 191-198.

Vitalo, A. G., Gorantla, S., Fricchione, J. G., Scichilone, J. M., Camacho, J., Niemi, S. M., ... Levine, J. B. (2012). Environmental enrichment with nesting material accelerates wound healing in isolation-reared rats. Behavioural Brain Research, 226, 606-612.
Vollmayr, B., Bachteler, D., Vengeliene, V., Gass, P., Spanagel, R., \& Henn, F. (2004). Rats with congenital learned helplessness respond less to sucrose but show no deficits in activity or learning. Behavioural Brain Research, 150, 217-221. doi:10.1016/S01664328(03)00259-6

Vollmayr, B., \& Henn, F. A. (2001). Learned helplessness in the rat: Improvements in validity and reliability. Brain Research Protocols, 8, 1-7.

Wainwright, P. E., Lévesque, S., Krempulec, L., Bulman-Fleming, B., \& McCutcheon, D. (1993). Effects of environmental enrichment on cortical depth and Morris-maze performance in B6D2F2 mice exposed prenatally to ethanol. Neurotoxicology and Teratology, $15,11-20$.

Walf, A. A., \& Frye, C. A. (2007). The use of the elevated plus maze as an assay of anxiety-related behavior in rodents. Nature Protocols, 2, 322-328.

Weary, D. M., \& Fraser, G. (1998). Replication and pseudoreplication: A comment on Phillips (1998). Applied Animal Behaviour Science, 11, 181-183.

Winocur, G. (1998). Environmental influences on cognitive decline in aged rats. Neurobiology of Aging, 19, 589-597.

Winter, C., Vollmayr, B., Djodari-Irani, A., Klein, J., \& Sartorius, A. (2011). Pharmacological inhibition of the lateral habenula improves depressive-like behavior in an animal model of treatment resistant depression. Behavioural Brain Research, 216, 463465. doi:10.1016/j.bbr.2010.07.034 